\title{
Identificación de larvas productoras de miasis obtenidas del cepario de la Universidad Colegio Mayor de Cundinamarca con importancia en salud pública
}

\author{
Identification of Myasis-producing Larvae from the Universidad Colegio \\ Mayor de Cundinamarca
}

Páez Díaz Ruth ${ }^{1}$, Villa Arteta Luisa Carolina ${ }^{1}$

\section{Resumen}

Las miasis son infestaciones parasitarias en humanos y animales causadas por estadios larvarios de moscas; son de distribución mundial y son una enfermedad frecuente en nuestro medio. En la literatura solo existen algunos casos reportados; por lo cual, su verdadera incidencia es difícil de establecer debido al sub-registro y ausencia de tipificación de larvas. Objetivo. Identificar, clasificar y caracterizar morfológicamente las larvas causantes de miasis como base para futuras aplicaciones e intervenciones en salud pública. Materiales y métodos. Se analizaron 262 larvas obtenidas del cepario de la Universidad Colegio Mayor de Cundinamarca que se encontraban almacenadas sin ningún tipo de identificación, organización e historia. Resultados. Se realizó la identificación con estereoscopio y claves dicotómicas de las familias Cuterebridae, Oestridae y Calliphoridae. Las especies encontradas son asociadas a diferentes tipos de miasis humana y animal, entre ellas; Dermatobia hominis, Dermatobia cyaniventris, Oestrus ovis, Cochliomyia hominivorax y Lucilia sp. Discusión. Las formas más comunes de este parasitismo son cavitarias y forunculares; causadas por Dermatobia hominis y Cochliomyia hominivorax, sin embargo, éstas no son de reporte obligatorio ante los servicios médicos humanos. Por lo tanto, es indispensable la información y capacitación de profesionales de la salud para la correcta conservación, identificación y reporte de las larvas extirpadas en pacientes con miasis, hasta el punto de ser considerada una práctica de rutina en el diagnóstico clínico.

Palabras claves: Miasis, Dermatobia hominis, Dermatobia cyaniventris, Oestrus ovis, Cochliomyia hominivorax, Lucilia sp., salud pública.

\section{Abstract}

Myiasis is the parasitic infestation of the body in humans and animals caused by larval stages of flies; such diseases are worldwide distributed and they are frequent in our environment. In the literature, there are only a few reports; therefore, its real incidence is difficult to be established due to subrecorded cases and absence of larval typing. Objective. To identify, classify and morphologically characterize myasis-producing larvae of importance in public health. Material and methods. 262 larvae were analysed, obtained from the Universidad Colegio Mayor de Cundinamarca that were stored without any identification, organization and history. Results. Larvae were identified

1. Programa de Bacteriología y Laboratorio Clínico, Facultad de Ciencias de la Salud, Universidad Colegio Mayor de Cundinamarca, Bogotá D.C., Colombia 
using a stereomicroscope and morphology was based on dichotomous keys of the Cuterebridae, Oestridae and Calliphoridae families. The species found are associated with different types of myiasis, including; Dermatobia hominis, D. cyaniventris, Oestrus ovis, Cochliomyia hominivorax, C. macellaria and Lucilia spp. Discussion. As a conclusion, we found that cavitary and foruncular were the most common forms of this parasitism in the collection from the Universidad Colegio Mayor de Cundinamarca. and that Dermatobia hominis and Cochliomyia hominivorax were the main involved species; however, these are not mandatory reporting species for medical services. Therefore, generating information about preservation, identification and recording of myasisproducing larvae, as well as training of professionals in public health might be considered as a routine practice for an accurate clinical diagnosis.

Keywords: Myasis, Dermatobia hominis, Dermatobia cyaniventris, Oestrus ovis, Cochliomyia hominivorax, Lucilia sp., Public Health.

\section{Introducción}

Las miasis son infestaciones parasitarias en humanos y otros animales vertebrados causadas por estadios larvarios de algunas especies de moscas del orden Diptera que al menos en un periodo de su desarrollo se alimentan de tejidos vivos o muertos, órganos, líquidos corporales o sustancias de alimento ingeridos por el hospedero, caracterizándose por su rápida y agresiva evolución $(1,2)$.

Sus manifestaciones clínicas se presentan según las especies de dípteros que actúan como el agente etiológico y el órgano o tejido infestado. El pronóstico está directamente relacionado con la duración, localización de la lesión y el estado de salud del paciente, siendo en la mayoría de casos-auto limitadas ya que las larvas abandonan el hospedero antes de pupar (3).

Pueden clasificarse según la localización anatómica del parásito en el hospedero (foruncular, cutánea, cavitarias, e intestinal) o según sus características de alimentación de la larva (obligatoria, facultativa y accidental).

Esta infestación es casi siempre accidental en humanos, pero es principalmente una plaga en animales domésticos y de producción. Las especies más frecuentes implicadas en miasis son Derma- tobia hominis, Cochliomyia hominivorax, Lucilia sericata, Oestrus ovis, Wohlfahrtia magnifica, Cordylobia anthropophaga y Chrysomya bezziana $(1,2,4)$. De manera que, la mayoría de miasis en humanos pueden ser causadas por especies oportunistas, en las que la infestación depende de varios factores: 1) la presencia de moscas hembras grávidas en busca de un sustrato de ovoposición; 2) excesiva exposición de un hospedero potencial susceptible a las moscas que causas miasis por deficientes condiciones de higiene e incorrecto tratamiento de heridas; 3) distribución geográfica; 4) época del año, particularmente en verano; 5) personas con movilidad disminuida, inconscientes, lesiones expuestas, halitosis, incontinencia y heridas supurativas que actúan como sustratos de atracción para la ovoposición (5-7). No obstante, en animales domésticos y de producción, los casos de miasis están asociados principalmente con condiciones y actividades de manejo, tipo de producción o susceptibilidad de la raza.

A pesar de que algunos estudios fisiológicos, bioquímicos e inmunológicos de los insectos de importancia médica y veterinaria, especialmente las moscas, se han llevado a cabo en la última década (8), teniendo en cuenta que las miasis son de distribución mundial y no es una enfermedad infrecuente en nuestro medio, en la literatura lo- 
cal solo existen algunos casos reportados a nivel cutáneo, ótico, nasofaríngeo y nasosinusal, por lo cual su verdadera incidencia es difícil de establecer; situación aumentada por el sub-registro de la enfermedad y ausencia de tipificación de las larvas $(9,10)$.

Las moscas responsables de esta patología presentan más especies y mayor abundancia en las regiones tropicales y subtropicales por su preferencia a ambientes húmedos y cálidos, considerándose endémicas en países de América Latina, Oriente Medio y Asia (11).

Además, son los insectos que generan mayor perjuicio a nivel mundial en salud pública y perdidas económicas considerables, afectando al ganado, la producción de leche y causando daños directos en el peso y fertilidad, lo que influye de forma negativa en la calidad de la piel de los mismos $(4,12)$.

Las miasis son más comunes en los países en desarrollo de América Latina, Oriente Medio y Asia; sin embargo, también se pueden presentar en países desarrollados por el aumento de inmigración $\mathrm{y}$ viajes a países endémicos $(5,13,14)$.

En Colombia, Soriano-Lleras y Osorno-Mesa (15) realizaron una recopilación de datos históricos de infestaciones causadas por artrópodos, en la que describen algunos casos de miasis. En 1991, Valderrama (16) publica una precisa revisión sobre la biología, la epidemiología y la patogenia de las miasis en humanos.

Se han publicado trabajos de revisión en el área veterinaria, varios reportes de caso de especies de importancia en salud pública y trabajos de investigación $(11,17,18)$.

Adicionalmente, Pape y colaboradores (19) presentan una lista taxonómica de las especies de moscas de la superfamilia Oestroidea registradas en colecciones entomológicas para Colombia y la región neotropical, en el que seńalan la importan- cia potencial médica y sanitaria en el municipio de La Pintada (Antioquia, Colombia).

\section{Materiales y métodos}

Se analizaron 262 larvas productoras de miasis obtenidas del cepario de la Universidad Colegio Mayor de Cundinamarca, que no habían sido identificadas y se encontraban almacenadas, sin ningún tipo de clasificación y que fueron donadas por docentes y estudiantes a través de los ańos con fines académicos para educar a los estudiantes de Bacteriología y Laboratorio Clínico.

Teniendo en cuenta que el estado de conservación de las larvas es un determinante para la correcta identificación de las larvas del cepario, se hizo necesario exclusivo y estricto el control de los criterios de exclusión para el éxito de este estudio.

Las larvas del cepario fueron clasificadas y codificadas para su conservación en etanol al 70\%. Fueron excluidas diez larvas que no cumplían con los criterios, al presentar deterioro como estar incompletas, destruidas, perforadas y desecadas; los cuales impedían la apropiada identificación y caracterización, evitando así la posibilidad de errores durante su clasificación.

La identificación de las larvas se realizó a partir de su caracterización morfológica en 4x y 10x en estereoscopio por su diferenciación taxonómica, en compañía de una revisión bibliográfica de claves dicotómicas de la superfamilia Oestroidea, en especial las familias Calliphoridae, Cuterebridae y Oestridae.

Las características principales para su clasificación fueron a partir de sus espiráculos, peritrema, abertura del peritrema, tubérculos, ganchos bucales, tráqueas respiratorias, esqueleto cefalofaringeo y el número de segmentos.

Para la identificación de Cochliomyia hominivorax se realizó la disección de una larva para visualizar 
el esqueleto cefalofaringeo y las tráqueas respiratorias, observándose en el estereoscopio y microscopio 4x y 10x.

Las muestras de larvas son examinadas de forma individual en el estereoscopio, y de esta manera se clasifican de acuerdo a su estadio. Se realiza la comparación con las claves taxonómicas. Para algunas larvas en estadio uno y dos es necesario realizar una técnica de aclaración en $\mathrm{KOH}$, lo que permite observar detalladamente ciertas estructuras claves para precisar la identificación de las mismas y saber en qué estadio se dispersan las mismas.

Para realizar el aclarado de las larvas, se sumergen las piezas bucales y esqueletos cefalofaríngeos en un tubo de ensayo o caja de Petri con hidróxido de potasio en solución acuosa $(\mathrm{KOH})$ al 10\%.

Para el procesamiento de los huevos, se deben poner inmediatamente en etanol al $70 \%$, para fijarlos y almacenarlos. Para examinar las estructuras de los huevos, se observan al microscopio en 40x.

Los resultados obtenidos de la caracterización morfológica fueron clasificados de acuerdo a las claves dicotómicas de identificación del Manual de la OIE sobre animales terrestres (8), Universidad de Alicante (9), Terry Whitworth (10), COPEG (20) y de la Universidad de Illinois, Chicago (12)

\section{Resultados}

De 262 las larvas del cepario de la Universidad Colegio Mayor de Cundinamarca, se identificaron en total 252 siguiendo las claves dicótomas seleccionadas previamente. Fueron excluidas 10 larvas sin poder lograr su identificación morfológica al cumplir los criterios de exclusión.

Se identificaron 20 larvas de tercer estadio (L3) de D. hominis; su caracterización y clasificación morfológica de se realizó a partir de su parte anterior en presencia de dos ganchos bucales de fijación (ver Figura 1).

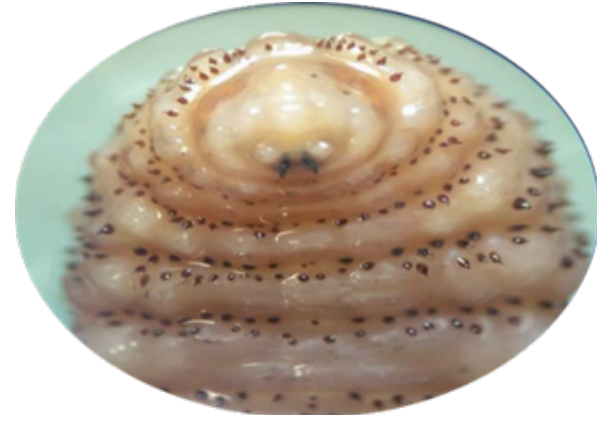

Figura 1. Vista ventral de larva de tercer estadio de Dermatobia hominis. a. Parte anterior primeros segmentos de la parte anterior con corona de espinas y ganchos bucales.

Se caracteriza por ser de contextura robusta con una reducción desde el segmento cefálico hasta el sexto segmento y ligeramente decreciente hacia el extremo posterior en los últimos tres segmentos; la longitud es de $1.0-2.0 \mathrm{~cm}$ y el ancho de $0,4-1,0 \mathrm{~cm}$.

La larva en estadio L3 usualmente es de $1,5 \mathrm{~cm}$ de longitud y $0.8 \mathrm{~cm}$ de ancho; las larvas al inicio de este estadio son de color blanco cremoso y la larva madura presenta una coloración ligeramente rojiza. Los segmentos dos al siete están acompañados con dos o tres hileras de espinas (ganchos de quitina) (ver Figura 2).

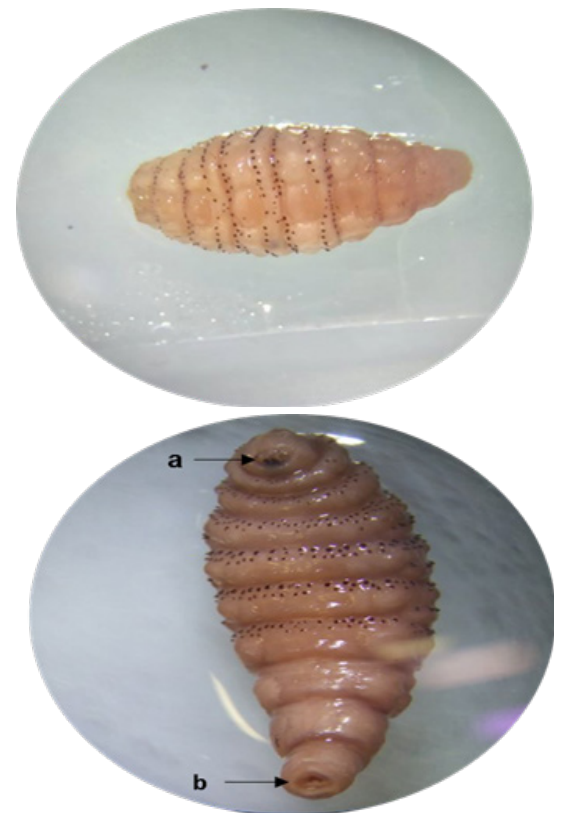

Figura 2. Vista dorsal y ventral de larva de tercer estadio de Dermatobia hominis. a. Corona de espinas en primer segmento con ganchos bucales en la parte anterior, $\boldsymbol{b}$. espiráculos posteriores. 
De este modo, se identificación 22 larvas de tercer estadio (L3) de Dermatobia cyaniventris, sin embargo; al pertenecer al género Dermatobia spp., presenta las características morfológicas típicas, con la especialidad de poseer dos espiráculos desarrollados en su extremo anterior, alcanzando en su tercer estadio larvario $0.8-3.0 \mathrm{~cm}$ de largo por 0.4 $1.0 \mathrm{~cm}$ de ancho (ver Figura 3 y Figura 4).

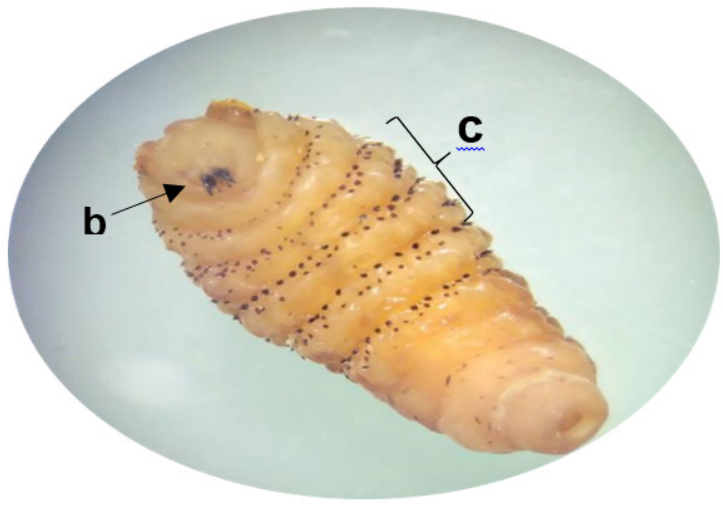

Figura 3. Vista ventral de larva de tercer estadio de Dermatobia cyaniventris. a. Espiráculos anteriores desarrollados, $\boldsymbol{b}$. Ganchos bucales, $\boldsymbol{c}$. Corona de espinas.
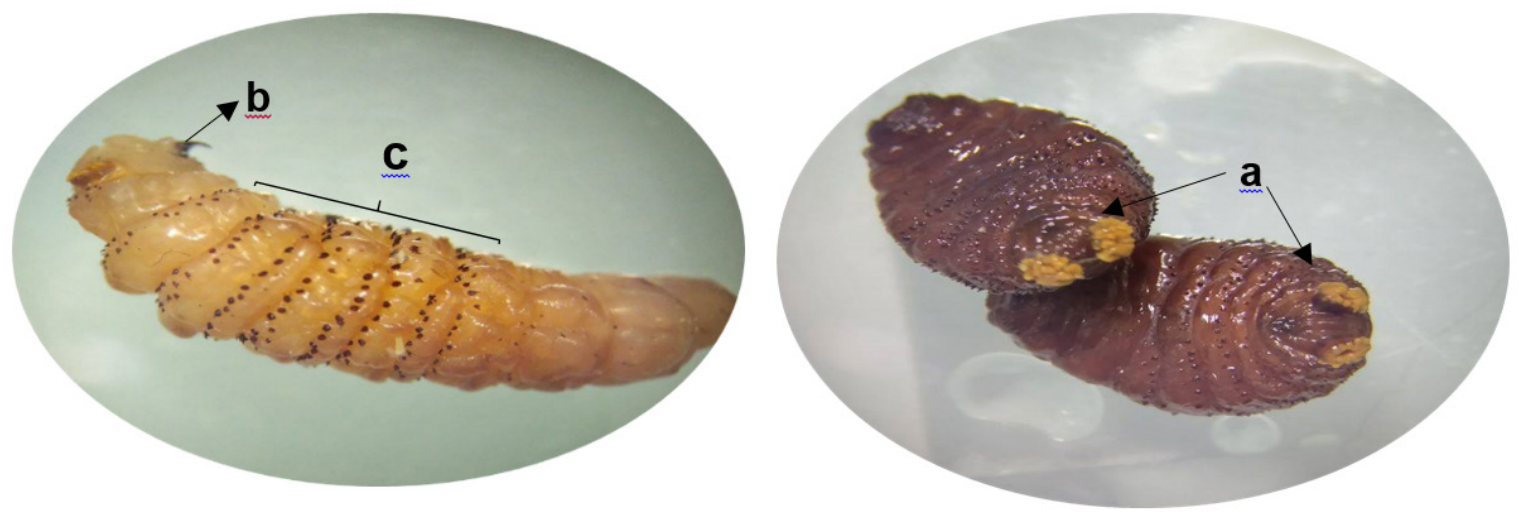

Figura 4. Vista lateral y ventral de larva de tercer estadio de Dermatobia cyaniventris. $\boldsymbol{a}$. Espiráculos anteriores desarrollados, $\boldsymbol{b}$. ganchos bucales, $\boldsymbol{c}$. Corona de espinas.

En cuanto a las larvas de O. ovis se identificaron 4 en estadio larvario (L3). La L3 se caracteriza por el cuerpo robusto cuadrangular mamelonado, dividido en once a doce segmentos cubiertos de espinas. Su longitud promedio es de 1,7-2,2cm y $0,7-0,8 \mathrm{~cm}$ de ancho.

El último segmento es bilobulado y muestra los espiráculos cerrados en forma de " $\mathrm{D}$ ", la parte anterior posee ganchos bucales en forma de cuernos, y en su extremo posterior presenta un doble abultamiento terminal con numerosas espinas (ver
Figura 5). Cada lóbulo está coronado de doce ganchos, su cara dorsal es convexa sin espinas, pero con franjas marrones en cada uno de los segmentos y su cara ventral es plana con tres a cuatro hileras de espinas (ver Figura 6).

Una característica propia de la familia Calliphoridae, es presentar espinas a través de todo el cuerpo de las larvas, agrava la acción parasitaria de las mismas en su paso por los conductos respiratorios del animal o humano, hasta la destrucción e invasión del tejido óseo. 

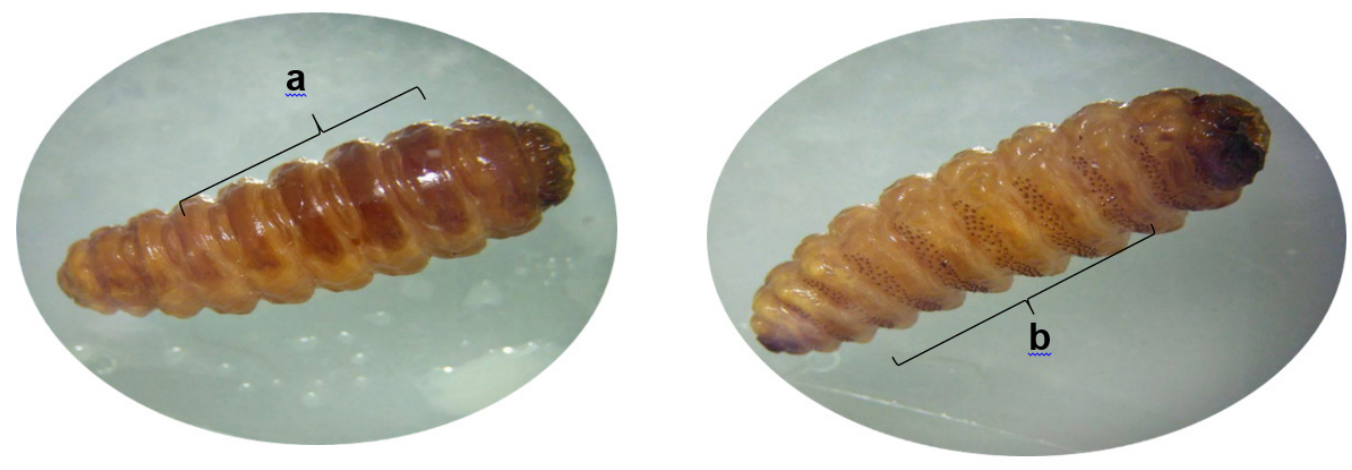

Figura 5.Vista dorsal de larva de tercer estadio de Oestrus ovis. a. Sin espinas con segmentos mamelonados de color marrón, $\boldsymbol{b}$. Vista ventral con espinas.
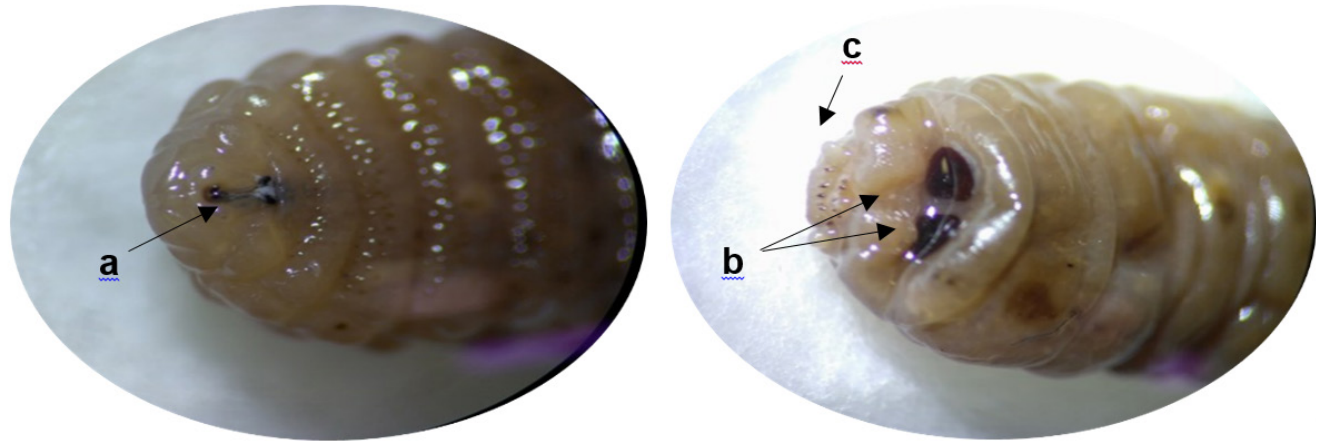

Figura 6.Vista ventral de larva de tercer estadio de Oestrus ovis. $\boldsymbol{a}$. Parte anterior con ganchos bucales y espinas, $\boldsymbol{b}$. Parte posterior con espiráculos cerrados en forma de "D”, $\boldsymbol{c}$. Tubérculos.

Así mismo, se identificaron 203 larvas de C. hominivorax; su clasificación se llevó a cabo por el aspecto de "Gusano tornillo" debido a su apariencia en espiral; es dividido en 12 segmentos, más angosto el segmento anterior y más ancho el posterior, cubiertos por tres a cuatro hileras de espinas pequeńas en cada segmento (ver Figura 7).

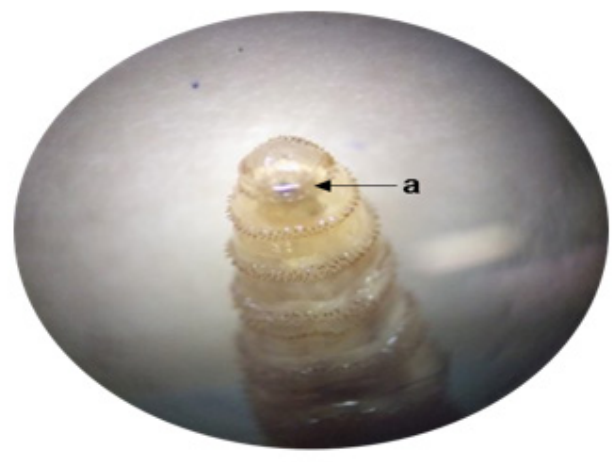

Adicional a lo anterior, 50 larvas pertenecían al estadio L1 (0,2-0,3cm de longitud y $0,1-0,2 \mathrm{~cm}$ de ancho). 53 larvas presentaban estadios larvarios (L2) en muda de transición a L3 con una longitud entre $0,2-0,5 \mathrm{~cm}$ y $0,1-0,3 \mathrm{~cm}$ de ancho (ver Figura 8).

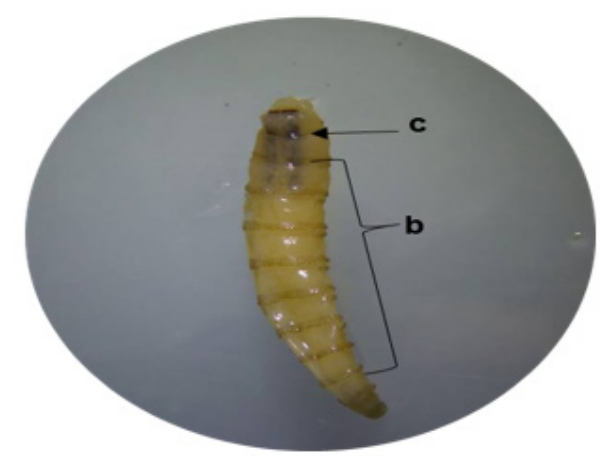

Figura 7. Vista ventral de larva de tercer estadio de Cochliomyia hominivorax. $\boldsymbol{a}$. Parte anterior con ganchos bucales, $\boldsymbol{b}$. Corona de espinas completas en los segmentos, $\boldsymbol{c}$. Tráqueas pigmentadas. 

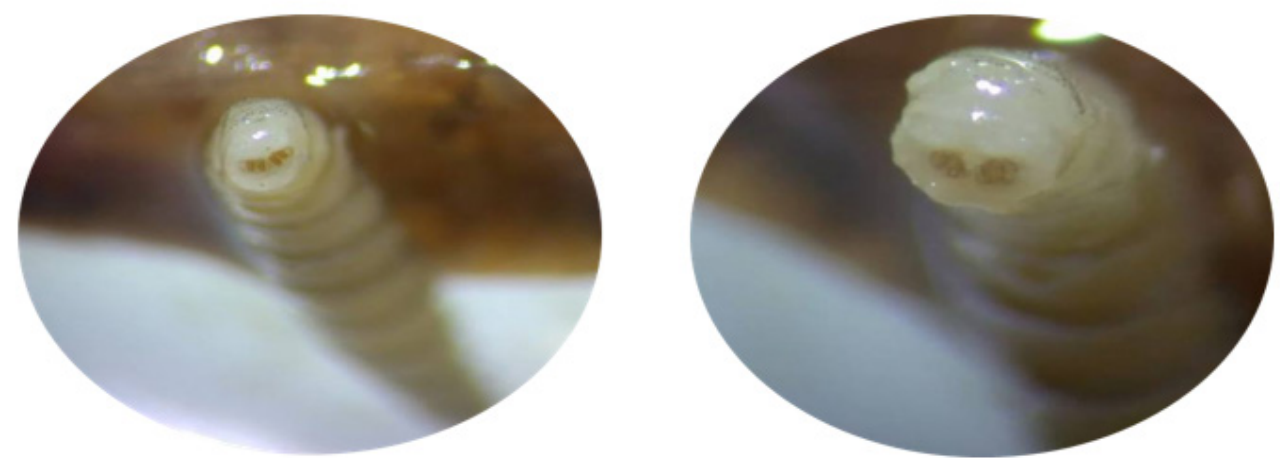

Figura 8. Espiráculos posteriores de larva en transición de primer a segundo estadio de Cochliomyia hominivorax.

Se clasificaron 100 larvas en tercer estadio (L3) tienen una longitud entre 1,0-1,6 cm y 0,2-0,4 cm de ancho. Se caracteriza por presentar en su segmento anterior dos ganchos bucales bien desarrollados y en su segmento posterior espiráculos respiratorios abiertos (ver Figura 9), (Figura 10), (Figura 11).
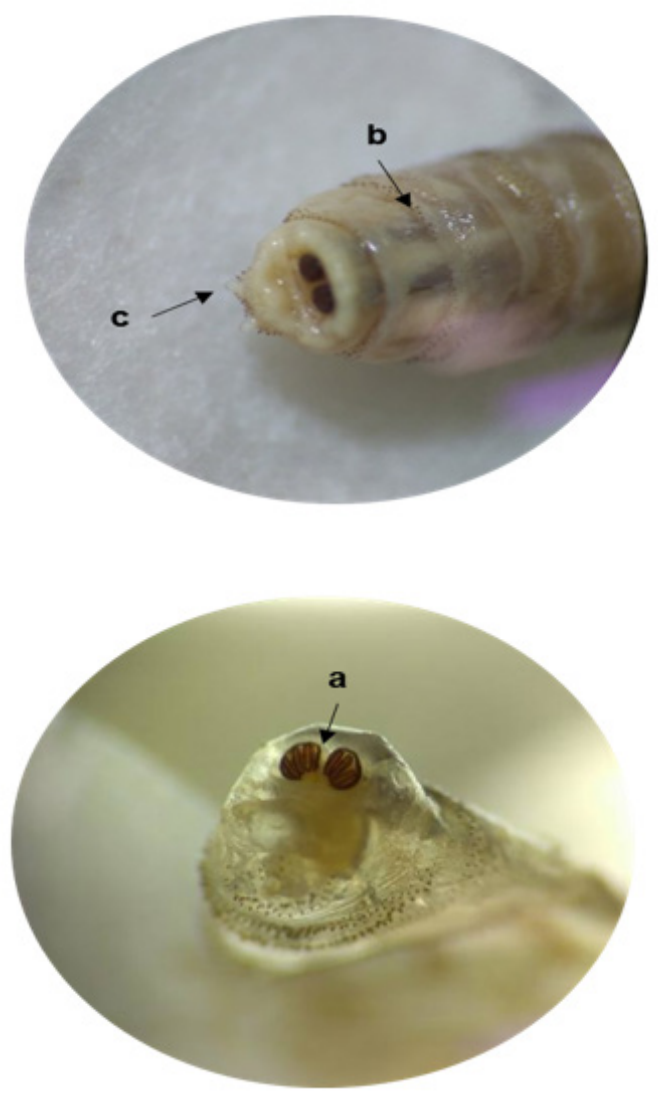

Figura 9. Cochliomyia hominivorax Cochliomyia hominivorax. $\boldsymbol{a}$. Parte posterior con espiráculos, $\boldsymbol{b}$. tráqueas pigmentadas, $\boldsymbol{c}$. tubérculos.

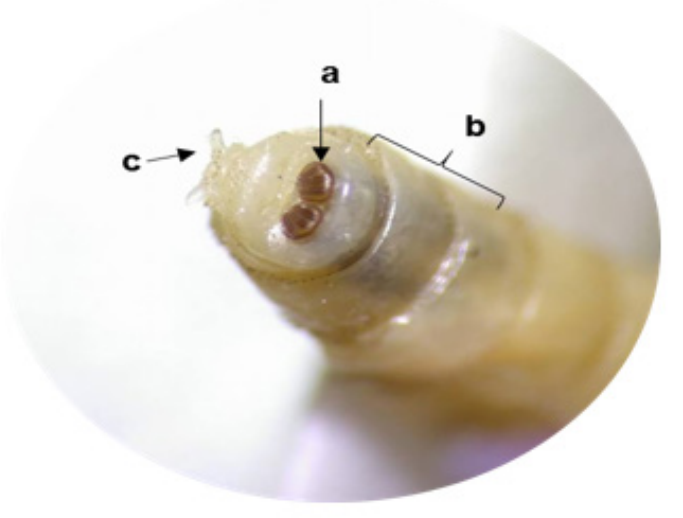

Figura 10. Espiráculos posteriores de larva de tercer estadio de Cochliomyia hominivorax. $\boldsymbol{a}$. Espiráculos, $\boldsymbol{b}$. tráqueas pigmentadas, $\boldsymbol{c}$. tubérculo anal.

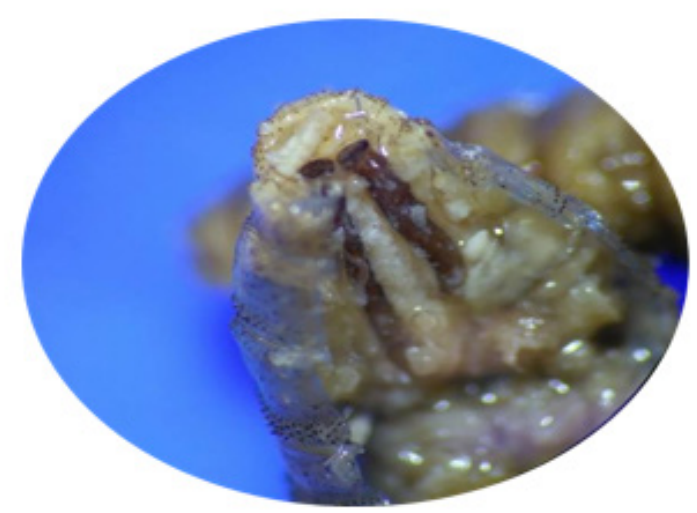

Figura 11. Disección de larva de tercer estadio de Cochliomyia hominivorax. Observación de tráqueas pigmentadas como característica morfológica en los últimos segmentos. 
Estos espiráculos son conectados con los anteriores por medio de dos tráqueas, ubicadas en los últimos segmentos, las cuales se encuentran en muy pigmentadas ofreciendo una característica distintiva para la identificación de esta especie; en su parte anterior se evidencia el esqueleto cefalofaríngeo (ver Figura 12 y (Figura 13).

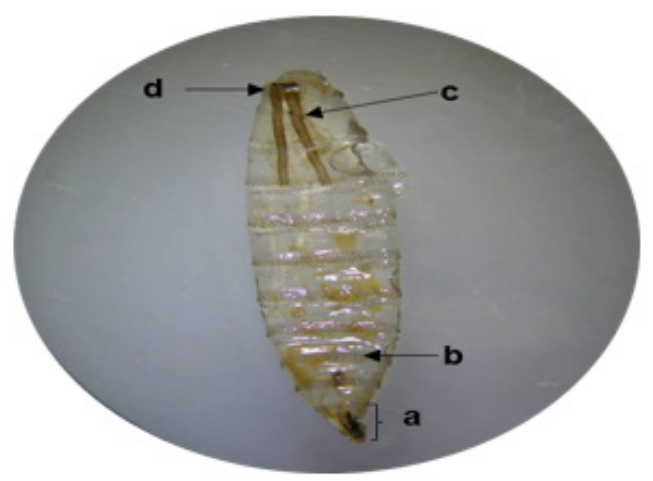

Figura 12. Larva de tercer estadio de Cochliomyia hominivorax aclarada con $\mathrm{KOH}$ al $10 \%$. a . Primer segmento torácico, $\boldsymbol{b}$.

Primer segmento abdominal, $\boldsymbol{c}$. Tráqueas, d. Espiráculo posterior.
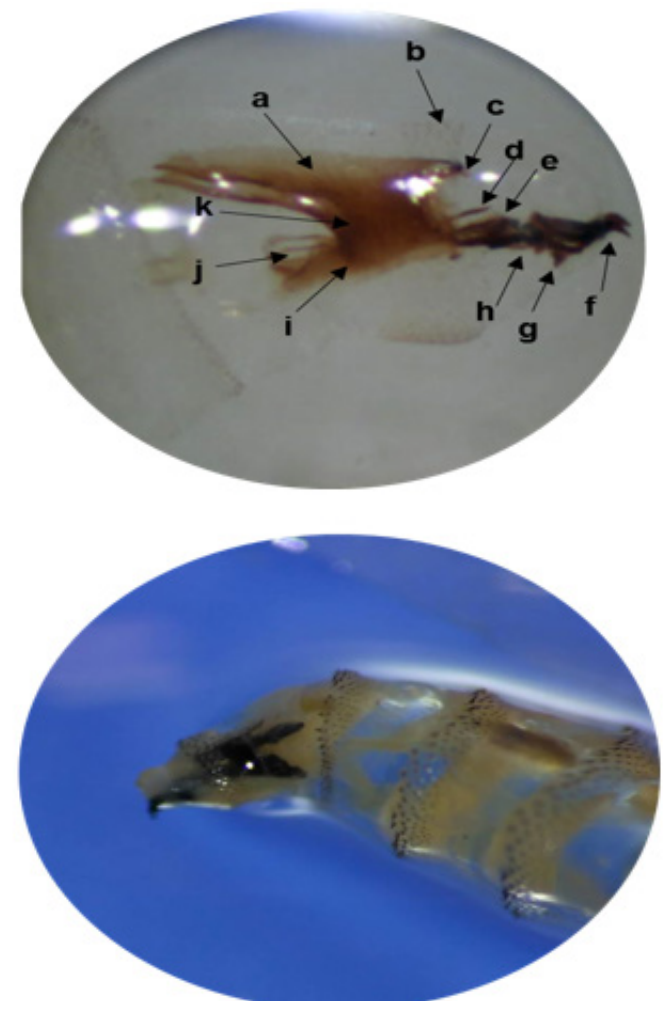

Figura 13. Esqueleto cefalofaríngeo de larva de tercer estadio de Cochliomyia hominivorax. a . Cuerno dorsal, $\boldsymbol{b}$. Espinas, $\boldsymbol{c}$. Puente dorsal, $\boldsymbol{d}$. Barra parastomal, $\boldsymbol{e}$. Hipo faringe, $\boldsymbol{f}$. Ganchos orales, $\boldsymbol{g}$. Esclérita dental, $\boldsymbol{h}$. Esclérita labial, $\boldsymbol{i}$. Cuerpo ventral, $\boldsymbol{j}$. Apodema ventral, $\boldsymbol{k}$. Fragma tentorial.
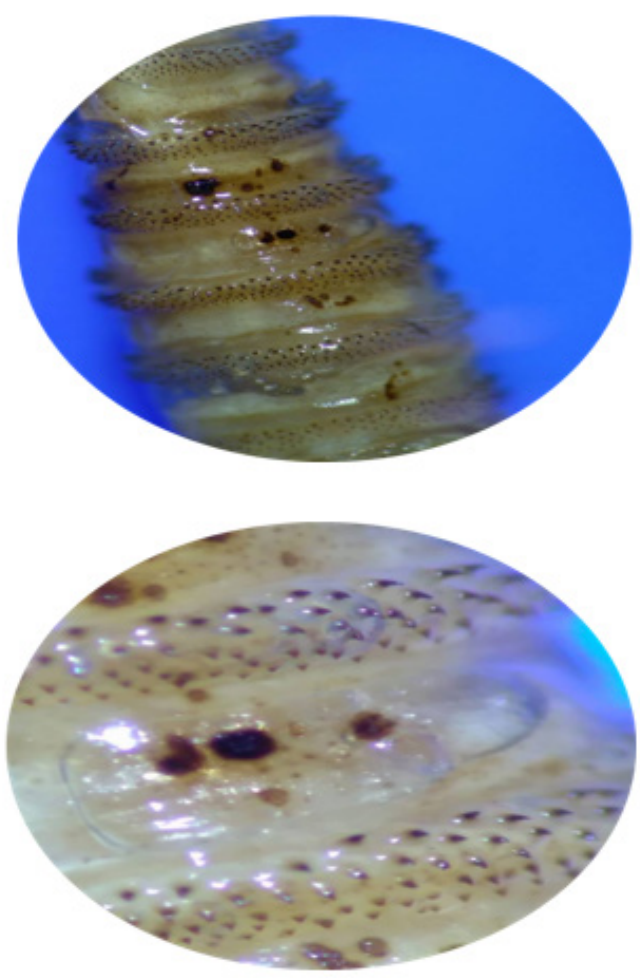

Figura 14. Espinas angulares de la punta de larva de tercer estadio de Cochliomyia hominivorax.

De igual modo, otra identificación adicional perteneciente al cepario, fueron los más de 600 huevos de moscas pertenecientes a $\mathrm{C}$. hominivorax caracterizados por ser de color blanco cremoso y presentar una sutura dorsal desde el opérculo hasta el extremo opuesto de forma completa (ver Figura 15), diferenciándolos de huevos de C. macellaria que presenta la sutura dorsal incompleta.

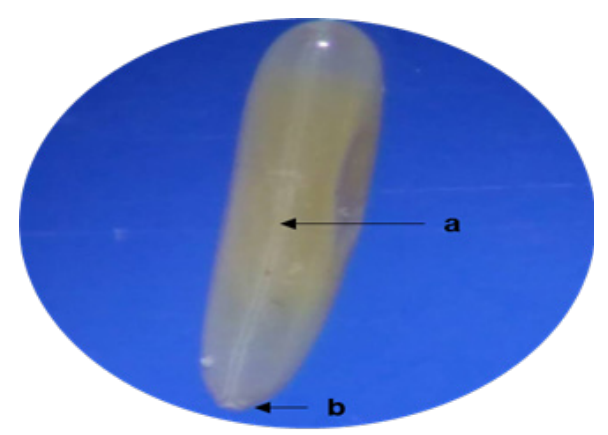

Figura 15. Huevo de Cochliomyia hominivorax. a. Sutura dorsal completa, $\boldsymbol{b}$. opérculo. 
Por último, se identificaron 3 larvas de Lucilia sp. con $1,3 \mathrm{~cm}$ de largo y $0,8 \mathrm{~cm}$ de ancho; caracterizadas por presentar tuberosidades bien detalladas y poseer un botón en el peritrema completo delgado, a diferencia de C. hominivorax que presenta un peritrema incompleto grueso (ver Figura $16 \mathrm{y}$ Figura 17).

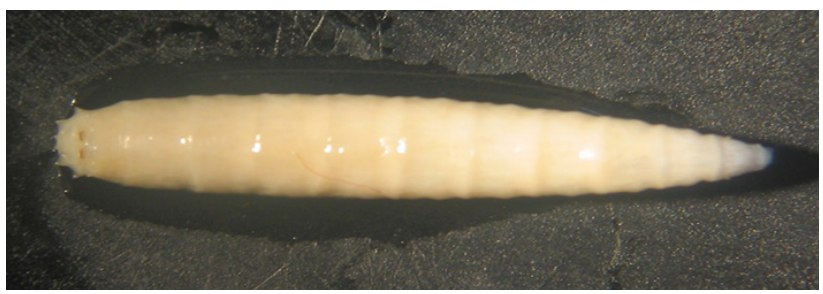

Figura 16. Vista dorsal de larva de tercer estadio de Lucilia sp.

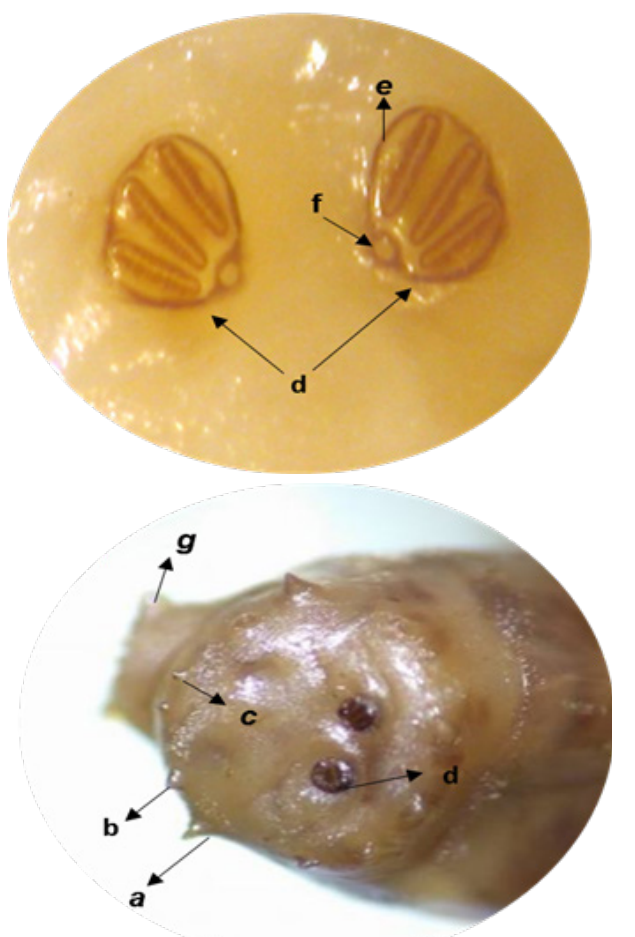

Figura 17. Vista dorsal de larva de tercer estadio de Lucilia sp. $\boldsymbol{a}$. Tubérculo externo $\boldsymbol{b}$. Tubérculo medio $\boldsymbol{c}$. Tubérculo interno $\boldsymbol{d}$. Espiráculos $\boldsymbol{e}$. Peritrema $f$. Botón respiratorio $g$. Tubérculos anales.

En el cepario de la Universidad Colegio Mayor de Cundinamarca se encontraron las siguientes especies: D. hominis, D. cyaniventris, O. ovis, Lucilia spp. y C. hominivorax (ver Tabla 1) con sus mediciones (ver Tabla 2).
Tabla 1. Identificación estadios larvales de miasis del cepario de la Universidad Colegio Mayor de Cundinamarca.

\begin{tabular}{|c|c|c|c|c|c|}
\hline \multirow{2}{*}{$\begin{array}{l}\text { Género y } \\
\text { Especies }\end{array}$} & \multicolumn{3}{|c|}{ Estadios Larvarios } & \multirow{2}{*}{ Huevos } & \multirow{2}{*}{$\begin{array}{c}\text { Total, } \\
\text { especies }\end{array}$} \\
\hline & L1 & L2 & L3 & & \\
\hline $\begin{array}{l}\text { Dermatobia } \\
\text { hominis }\end{array}$ & 0 & 0 & 20 & 0 & 20 \\
\hline $\begin{array}{l}\text { Dermatobia } \\
\text { cyaniventris }\end{array}$ & 0 & 0 & 22 & 0 & 22 \\
\hline Oestrus ovis & 0 & 0 & 4 & 0 & 4 \\
\hline $\begin{array}{l}\text { Cochliomyia } \\
\text { hominivorax }\end{array}$ & 50 & 53 & 100 & $>600$ & 203 \\
\hline Lucilia sp. & 0 & 0 & 3 & 0 & 3 \\
\hline $\begin{array}{l}\text { Total, } \\
\text { identificadas }\end{array}$ & 50 & 53 & 149 & $>600$ & 252 \\
\hline
\end{tabular}

Tabla 1. Identificación estadios larvales de miasis del cepario de la Universidad Colegio Mayor de Cundinamarca.

\begin{tabular}{|l|l|l|l|r|}
\hline \multirow{2}{*}{ Género y especies } & \multicolumn{2}{|c|}{ Largo $(\mathrm{cm})$} & \multicolumn{2}{c|}{ Ancho (cm) } \\
& Mínimo & Máximo & Mínimo & Máximo \\
\hline Dermatobia hominis & 1.0 & 2.0 & 0.4 & 0.9 \\
\hline Dermatobia cyaniventris & 0.8 & 3.0 & 0.4 & 1.0 \\
\hline Oestrus ovis & 1.7 & 2.2 & 0.7 & 0.8 \\
\hline $\begin{array}{l}\text { Cochliomyia } \\
\text { hominivorax L1 }\end{array}$ & 0.2 & 0.3 & 0.1 & 0.1 \\
\hline $\begin{array}{l}\text { Cochliomyia } \\
\text { hominivorax L2 }\end{array}$ & 0.2 & 0.5 & 0.1 & 0.3 \\
\hline $\begin{array}{l}\text { Cochliomyia } \\
\text { hominivorax L3 }\end{array}$ & 1.0 & 1.6 & 0.2 & 0.4 \\
\hline Lucilia sp. & 1.3 & 1.3 & 0.8 & 0.8 \\
\hline
\end{tabular}

Tabla 2. Medidas de las larvas del cepario de la Universidad Colegio Mayor de Cundinamarca.

\section{Discusión}

El presente estudio logra la identificación y caracterización morfológica de larvas de moscas causantes de miasis presentes en el cepario de la Universidad Colegio Mayor de Cundinamarca; se caracterizaron 252 larvas en adecuada conservación, excluyendo las que se encontraban en deterioro.

A lo largo de esta investigación se logró demostrar que a partir del género y especie identificado depende su clasificación de productor de miasis o 
no, de modo que, las cinco especies identificadas, Dermatobia hominis, Dermatobia cyaniventris, Oestrus ovis, Cochliomyia hominivorax y Lucilia sp., son asociadas sin duda alguna como larvas productoras de diferentes tipos de miasis en humanos $\mathrm{y}$ animales.

Es necesario resaltar que, las claves de identificación taxonómica fueron elegidas por presentar la descripción y caracterización de las estructuras representativas de cada especie como, espiráculos posteriores y anteriores, ganchos bucales, esqueleto cefalofaringeo, tráqueas respiratorias, número de segmentos, espinas y contextura física. Algunas de estas claves presentan gráficos y fotografías como guía para su caracterización.

Los principios de estos resultados, justifican el uso correcto de dichas claves, llegando hasta la identificación de especies. Se presentan las fotografías con las estructuras representativas y su caracterización morfológica de las larvas. De esta manera, se realizó una revisión bibliográfica de artículos de casos clínicos de miasis en humanos y animales causados por las especies de larvas presentes en el cepario de la Universidad Colegio Mayor de Cundinamarca.

Por consiguiente, la identificación de C. hominivorax según las claves utilizadas, se diferencia de C. macellaria por sus espinas bífidas, espiráculo anterior, cefaloesqueleto con escléritas labiales más pequeñas, espiráculos posteriores más pequeños y redondeados (8-10,20). Además, es de resaltar, que el huevo es aparentemente proveniente de una queresa de C. hominivorax, fue diferenciado de C. macellaria por su sutura dorsal longitudinal incompleta $(20)$

En adición, la diferenciación de especies del género Dermatobia, se realizó a partir de sus espiráculos anteriores, que en el caso de $\mathrm{D}$. cyaniventris los posee desarrollados a diferencia de D. hominis $(9,10)$.

De los casos reportados, la miasis cutánea junto a la de heridas, son las presentaciones clínicas que presentan mayor frecuencia, incluidas las miasis forunculares y migratorias, siendo D. hominis y C. anthropophaga los principales causantes.

El diagnóstico diferencial entre D. hominis y C. anthropophaga radica en que la primera es la mosca económicamente más importante al ser endémica en la región tropical de Centro y Sur América con difícil control y erradicación debido a su fenómeno único de ovoposición conocido como foresis; mientras que la segunda, es autóctona del sur del Sahara y África Central, con tres estrías onduladas oscuras en torno a los dos espiráculos posteriores $(4,14)$.

Las formas más comunes de miasis humana son la cavitaria y foruncular, esta última es a menudo causada por la infestación obligatoria de larvas subcutáneas por la mosca D. hominis y C. hominivorax en las zonas subtropicales y tropicales de América $(3,16,21)$.

Es de particular importancia la especie cosmopolita Oestrus ovis, cuyas larvas se desarrollan en las cavidades nasales y sinusales de ovinos y caprinos. A pesar de su alta especificidad, se han reportado varios casos incidentales en humanos como oftalmomiasis y miasis oral (21-23). Este parásito también ha sido encontrado en perros y gatos, de modo que es considerada una zoonosis accidental. Sin embargo, los casos de este parasitismo no son de reporte obligatorio en Colombia y América Latina $(18,24)$.

Varios estudios confirman que hay un riesgo alto de infestación de animales y humanos susceptibles, situados en determinadas latitudes y su relación con el tamańo de la población como en el caso de Colombia (18).

Es de suma importancia el diagnóstico preciso, identificación y tipificación de estas larvas con el objeto de conocer el comportamiento de esta patología en nuestro medio e igualmente con el propósito de identificar el tipo de mosca que está afectando no 
solo a población humana vulnerable, sino que también afecta grandemente a las industrias ganaderas. Todo ello con el ánimo de generar políticas de salud pública que detengan la migración de estos insectos y el daño que estos causan al ecosistema con el fin de lograr la erradicación de la enfermedad (21).

En gran parte de América se considera que C. hominivorax es una de las principales plagas de artrópodos antecedida por las garrapatas. En la actualidad, 330.570 de personas (25) y más de 515 millones de animales (26), están en riesgo de ser infectados por C. hominivorax.

Debe considerarse la erradicación del Gusano Barrenador del Ganado Primario (C. hominivorax) y el Gusano Barrenador del Ganado Secundario (C. masellaria), diferenciándose por el tipo de tejido del cual se alimentan. En el caso del primario, consume todos los tejidos vivos de animales de sangre caliente y hombre; mientras que el secundario tiene una alimentación necrobiontófoga o mixta.

Algunos de los casos de miasis humana reportados en Colombia han sido en agricultores, pero es probable que exista un subregistro de casos en el país y por lo tanto la incidencia de la enfermedad es subestimada (19). Por lo que las heridas iatrogénicas o prácticas de manejo injuriantes en animales de producción favorecerían el desarrollo de miasis traumática y aumentarían el riesgo de presentación en humanos. El riesgo de miasis foruncular en humanos a partir de animales domésticos como reservorio no ha sido evaluado en Colombia (27).

La miasis cavitaria es zoonótica y por lo tanto de gran importancia para la salud pública, ya que se relaciona de forma directa con los hábitos higiénicos deficientes de las personas, siendo estas las más propensas a tener lesiones cutáneas o enfermedades sistémicas, y son, en consecuencia, altamente susceptibles a la infestación (28).

Adicionalmente, debería existir una estrecha cooperación entre los veterinarios de los servicios de asistencia humana y los bacteriólogos de los servicios de salud pública en Colombia durante la realización de estudios epidemiológicos de miasis cavitarias, facultativas zoonóticas y accidentales.

Ahora bien, tanto en la literatura médica como veterinaria, la epidemiología de las enfermedades infecciosas y parasitarias ha sido relacionada con condiciones de pobreza (29). Pero, en los últimos años, ha existido una tendencia creciente hacia la privatización o disminución de los presupuestos de los servicios médicos y veterinarios estatales en los países en vías de desarrollo $(13,18)$; por lo que la disponibilidad y accesibilidad a estos se encuentran restringidas para la población de más escasos recursos.

En los países en vías de desarrollo y particularmente en Colombia, la información del sector público sobre la incidencia e impacto de las miasis en términos de mortalidad, morbilidad, costos de producción, resistencia a antiparasitarios, entre otros, se encuentra limitada. De hecho, las miasis cavitarias no son de reporte obligatorio ante los servicios médicos estatales en Colombia y puede existir un número elevado de casos humanos no registrados.

Colombia como país miembro de la Organización Mundial de Sanidad Animal, acoge la lista de enfermedades, infecciones e infestaciones de la lista vigente de la OIE. Por esto, la Resolución 3714 de 2015 establece que las miasis en varias especies por Chrysomya bezziana y Cochliomyia hominivorax son de declaración obligatoria ante el Instituto Colombiano Agropecuario, sin embargo, en el sistema de vigilancia y reporte, es limitado es registro (29).

Sin lugar a dudas este problema ha minimizado la incidencia real de esta parasitosis hasta el punto de considerarse no necesario su reporte ante las entidades sanitarias.

Las pérdidas económicas en la industria están representadas por el costo de los medicamentos y servicios veterinarios, por el tiempo requerido para 
recuperar el nivel óptimo productivo de los individuos parasitados, por la muerte de los animales y por los dańos causados al cuero.

El reporte obligatorio de los casos de miasis debería ser la base para un programa de vigilancia nacional que apoye a los servicios estatales de salud pública. De esta manera, se pueden prever los factores que podrían causar la emergencia o reemergencia de estas parasitosis en zonas donde no ha habido registro de miasis (30-38).

Debido a la baja cantidad de centros de salud con profesionales capacitados para la identificación, clasificación, caracterización morfológica y confirmación molecular de los estadios larvarios de moscas productoras de miasis, es de vital importancia generar estrategias de diagnóstico, control y prevención de la enfermedad con el fin de lograr la erradicación total de la enfermedad debido a su importancia en la salud pública de nuestro país $(28,31)$.

Es así que resulta prioritario informar y capacitar a profesionales de la salud humana y animal para el adecuado reconocimiento de los signos y síntomas de las miasis, su respectiva identificación larvaria y confirmación por dermatoscopia, con el fin de dar un diagnóstico oportuno y tratamiento apropiado para evitar secuelas. Por lo tanto, es indispensable la correcta conservación y posterior identificación de las larvas extirpadas en pacientes con miasis, hasta el punto de ser considerada una práctica de rutina en el diagnóstico clínico.

Bajo este esquema, la creación de conciencia y la educación en salud de las comunidades urbanas y rurales pobres, así como la mejora del registro, resulta un eslabón esencial para tomar medidas más adecuadas para el control y prevención de esta parasitosis.

\section{Conflicto de intereses}

Los autores declaran no tener ningún conflicto de intereses.

\section{Financiación}

Los autores declaran no haber recibido financiación para la realización de este trabajo.

\section{Responsabilidades éticas}

Protección de personas y animales. Los autores declaran que para esta investigación no se han realizado experimentos en seres humanos ni en animales.

\section{Referencias}

1. Cepeda R, Angulo C, Ramirez - Orduña R. Ecobiology of the sheep nose bot fky (Oestrus ovis): a review. Revista Médica Veterinaria. 2011; 162(11): p. 503-507.

2. Everett E, DeVillez RL, Lewis CW. Cutaneous myiasis due to Dermatobia hominis. ARchivies of Dermatology. 1977; 113(8): p. 1122.

3. Ferraz A, De Almeida D, De Jesús D, Rotatori G, Nunes R, Proenca B, et al. Neotropical entomology epidemiological study of myiases in the Hospital do Andarai Rio de Janeiro. ncluding Reference to an Exotic Etiological Agent. 2011; 40: p. 393-397.

4. Rancesconi F, Lupi O. Myiasis. Clinical Microbiology Reviews. $2012 ;$; p. 79-105.

5. Dondero TJ, Schaffner W, Athanasiou R, Maguire W. Cutaneous myiasis in visitors to Central America. Southem Medical Journal. 1979; 72(12): p. 1508-1511.

6. Guimaraes J, Papevero N, Prado A. As miíases da regiao Neotropical. Revista Bras Zool. 1983; 1: p. 239-416.

7. Neves D. Parasitología Humana Sao Pablo: Atheneu; 1995.

8. OIE. Miasis por Cochliomyia hominivorax y miasis por Chrysomya bezziana. In Manual de la OIE sobre animales terrestres.; 2008. p. Capitulo 2.1.10.

9. Alicante Ud. CLave de Identificación de las larvas de tercer estadio que causan miasis en animales domesticos. Departamento de Ciencias Ambientales y Recursos Naturales. .

10. Whitworth T. Claves para Géneros y especies de moscas califóridas (Díptera: Calliphoridae) de América al norte de México. Proc. Entomol. Soc. Wash. 2006; 108(3): p. 689-725.

11. Becerra F, Gustavo e, Cortés Vecino JA, Villamil Jiménez LC. Factores de riesgo asociados a la miasis por Cochliomyia hominivorax en fincas ganaderas de Puerto Boyacá (Colombia). Universidad de Zulia: Revista Científica. 2000; 19(5): p. 460465 .

12. Debang L, Greenberg B. Inmature stages of some flies of forensic importane, Departament of Biological Sciences, Uni- 
versity of Illinois at Chicago. Entomological Society of America. ; 82(1989).

13. De Groote T, de Paepe P, Unger JP. Colombia: In vivo test of health sector privitazation in the developing world. International Journal of Health Services. 2005; 35(1): p. 125-141.

14. Vaquero M, Azcue M. Miasis Dermatobia hominis adquirida durante un viaje a Argentina. Med. Clinica de Barcelona. 2008; 131(9).

15. Soriano-Lleras A, Osorno-Mesa E. Datos históricos de observaciones hechas en Colombia sobre artópodos molestos y patológicos para el hombre. Revista de la Facultad de MEdicina. 1963; 3: p. 3-27.

16. Valderrama R. Miasis y humanos. Universidad de Antioquia, IATREIA. 1991; 4(2): p. 70

17. Vilmaris M. Oestrus ovis (Díptera: Oestridae): un importante extoparásito de los ovinos en Cuba. Revista de Salud Animal. 2013; 35(2): p. 79-88.

18. Forero Becerra E. Miasis en salud pública y salud pública veterinaria. Sapuvet de Salud Pública. 2011; 2(2): p. 95-132.

19. Pape T, Wolff M, Amat E. Los califóridos, estridos, rinofóridos y sarcofágidos de colombia (Díptera: Calliphoridae, Oestridae, Rhinophoridae, Sarcophagidae). Biota Colombiana. 2004; 5(2): p. 201-208.

20. COPEG. Manual de Identificación de Gusano Barrenador del Ganado Cochliomyia hominivorax (Coquerel) Diptera: Calliphoridae y su diferenciación de otras especies causantes de miasis. Comisión México-americana para la erradicación del Gusano Barrenador del Ganado. 2008; MAyor.

21. Díaz J. The epidemiology, Diagnosis, Management, and Prevention of Ectoparasitic Diseases in Travelers. Journal of Travel Medicine. 2006; 13: p. 100-111.

22. Alcaide et al. Seasonal variations in the larval burden distribution of Oestrus ovis in sheep in the sourthwest of Spain. Vet. Parasitology. 2003;: p. 118,235-241.

23. Alcaide M, Reina D, Frontera E, Navarrete I. Epidemiology of Oestrus ovis (Linneo, 1761). Infestation in goats in Spain. Veterinary Parasitology. 2005; 130(3-4): p. 277-284.

24. Yepes GD, Sánchez Rodríguez J, De Mello Patiu C, Wolff Echeverri M. Sinatropía de Sarcophagidae (Diptera) en la Pintada, Antioquia-Colombia. RBT. 2013; 61(3).

25. Entorno Ganadero. Sitio Argentino de Producción Animal. [Online].; 2014 [cited 2017 Julio 13.

26. FAO. EL GUSANO BARRENADOR DEL GANADO. [Online]. [cited 2017 Mayo 12. Available from: http://www. fao.org/3/a-ai173s/ai173s02.pdf.

27. Cruz S, Méndez I. Foruncular myiasis Eco-epidemiological view of a case report. Elsevier. 2014.

28. Villamizar JR, Sandoval Ortiz GP. Miasis ótica. Colombia: Revista de Otorrinolaringología. 2000 Septiembre; 28(3): p. 203-206.

29. Rodríguez A. Enfermedades olvidadas: miasis. Revista Peruana de MEdicina Experimental y salud pública. 2006; 23(2): p. 1434 .

30. Sánchez- Sánchez R, Calderon - Arguedas O, Mora Brenes N, Troyo A. Miasis nosocomiales en América Latina y el Caribe: ¿Una realidad ignorada? Revista Panamericana de Salud Pública. 2014; 36(3): p. 201-205.

31. Zúñiga Carrasco I. Miasis: un problema de salud poco estudiado en México. Revista Enfermedades infecciosas pediátricas. 2009; 88(22).

32. Lumbreras y Polack. Primer caso peruano de oculomiasis producida por larvas de Oestrus ovis Linneo, 1758. Revista Médica Peruana 26. 1758;: p. 95-99.

33. Alcaide, Reina, Frontera y Navarrete. Epidemiology of Oestrus ovis (Linneo, 1761). Veterinary parasitology. 2005;: p. 277-284.

34. Beltrán M, Torres G, Segami H, Náquira C. Miasis ocular por Oestrus ovis. Revista Peruana de Medicina Experimental y Salud Pública. 2006; 23(1): p. 70-72.

35. Sukontason K, Methanitikorn, Sukontason, S. Piangjai, J. Olson. Clearin thecnique to examine the cephalopharyngeal skeletons of blow fly larvae. Journal of Vector Ecology. 2004; p. $192-195$.

36. FAO. Manual para el control de la mosca del Gusano Barrenador del Ganado. FAO. 1993; 1.

37. Corrales, L. C., et al. Bacterias anaerobias: procesos que realizan y contribuyen a la sostenibilidad de la vida en el planeta. 2015; Nova 13(24): 55-82.

38. Rodríguez, O. E., Andrade, W. A., Díaz, F. E., \& Moncada, B. Actividad antimicrobiana de líquenes de la cuenca alta del rio Bogotá. 2015; Nova, 13(23). 